\title{
X-RAY GRATING OBSERVATIONS OF RECURRENT NOVA T PYXIDIS DURING THE 2011 OUTBURST
}

\author{
Benjamin M. Tofflemire ${ }^{1}$, Marina Orio ${ }^{1,2}$, Kim L. Page ${ }^{3}$, Julian P. Osborne ${ }^{3}$, Stefano Ciroi $^{4}$, \\ Valentina Cracco ${ }^{4}$, Francesco Di Mille $^{4}$, and Michael Maxwell ${ }^{5}$ \\ ${ }^{1}$ Astronomy Department, University of Wisconsin-Madison, 475 N. Charter St., WI 53711, USA \\ 2 INAF-Osservatorio Astronomico di Padova, Vicolo dell'Osservatorio 5, I-35122 Padova, Italy \\ ${ }^{3}$ Department of Physics and Astronomy, University of Leicester, Leicester LE1 7RH, UK \\ ${ }^{4}$ Department of Physics and Astronomy, Padova University, Vicolo dell'Osservatorio 3, I-35122 Padova, Italy \\ ${ }^{5}$ Jeremiah Horrocks Institute, University of Central Lancashire, Preston PR1 2HE, UK \\ Received 2013 March 13; accepted 2013 October 23; published 2013 November 22
}

\begin{abstract}
The recurrent nova T Pyx was observed with the X-ray gratings of Chandra and XMM-Newton, 210 and 235 days, respectively, after the discovery of the 2011 April 14 outburst. The X-ray spectra show prominent emission lines of C, N, and O, with broadening corresponding to an FWHM of $\sim 2000-3000 \mathrm{~km} \mathrm{~s}^{-1}$, and line ratios consistent with high-density plasma in collisional ionization equilibrium. On day 210 we also measured soft X-ray continuum emission that appears to be consistent with a white dwarf (WD) atmosphere at a temperature $\sim 420,000 \mathrm{~K}$, partially obscured by anisotropic, optically thick ejecta. The X-ray continuum emission is modulated with the photometric and spectroscopic period observed in quiescence. The continuum at day 235 indicated a WD atmosphere at a consistent effective temperature of 25 days earlier, but with a lower flux. The effective temperature indicates a mass of $\sim 1 M_{\odot}$. The conclusion of partial WD obscuration is supported by the complex geometry of non-spherically symmetric ejecta confirmed in recent optical spectra obtained with the Southern African Large Telescope in November and December of 2012. These spectra exhibited prominent [O III] nebular lines with velocity structures typical of bipolar ejecta.
\end{abstract}

Key words: novae, cataclysmic variables - stars: individual (T Pyxidis) - X-rays: stars

Online-only material: color figures

\section{INTRODUCTION}

T Pyxidis is a recurrent nova ( $\mathrm{RN})$ with recorded outbursts in 1890, 1902, 1920, 1944, 1966, and 2011. Although models and observations indicate that all classical novae $(\mathrm{CNe})$ may be recurrent in nature (Robinson 1976; Ringwald et al. 1996), $\mathrm{RNe}$ compose a rare subset whose outbursts reoccur on human timescales (i.e., recurrence times $<100 \mathrm{yr}$ ). The mechanism powering novae has been established as CNO-cycle driven thermonuclear runaway (TNR) on the surface of a white dwarf (WD) in an accreting binary system. About a dozen RNe are known in the Galaxy with a handful in the LMC and M31 (Schaefer 2010).

Apart from the frequency of outbursts, $\mathrm{RNe}$ differ from $\mathrm{CNe}$ in their mass accretion rates and peak luminosities. Models reproducing the short recurrence times of RNe (e.g., Yaron et al. $2005)$ require the combination of a massive WD and large mass accretion rates $(\dot{M})$. Massive WDs require less accreted material to reach the critical pressure for TNR, and with a large $\dot{M}$, outburst events are predicted on decade-long timescales. The resultant outbursts consequently have less fuel, are generally less luminous, and have shorter decay times than $\mathrm{CNe}$ (Webbink et al. 1987).

Unlike several other RNe, T Pyx is a system with little apparent secondary evolution and hosts an $\mathrm{M}$ dwarf companion (Selvelli et al. 2008). It belongs to a small group of RNe including also two other objects, IM Nor and CI Aql, where outbursts develop more slowly than in most RNe (see Strope et al. 2010). Furthermore, its orbital period is suggested to be below the "period gap" (Uthas et al. 2010) that canonically marks a regime of gravitational radiation driven angular momentum loss with $\dot{M} \sim 10^{-10} M_{\odot} \mathrm{yr}^{-1}$ (Iben \& Fujimoto 2008). This estimate is two orders of magnitude less than inferred from observations
(Patterson et al. 1998) and much less than what is predicted for the short recurrence time observed.

T Pyx is also an enigmatic object because the mass ejected in each outburst seems to be of order $10^{-5} M_{\odot}$, too large to have been accumulated in the 12-44 yr between nova events. It has been speculated that the WD mass is eroded by a large amount in each eruption (Selvelli et al. 2008). These estimates may be inflated, however, given the evidence for bipolar outflows (Chesneau et al. 2011) and a low filling factor of ejected material (as little as 3\%; Shore et al. 2011)

Most intriguing is the increasing recurrence time. Before 1966, T Pyx had a mean recurrence time of $\sim 20 \mathrm{yr}$ and yet remained in quiescence for over $44 \mathrm{yr}$ till the 2011 outburst. A drop in the average $B$-band magnitude by $\sim 2$ mag in the past $120 \mathrm{yr}$ (Schaefer et al. 2010) has been argued to represent a decreasing accretion rate to explain the recurrence trend. The above characteristics beg the question of whether T Pyx is in a transient evolutionary state. Authors prior to the 2011 outburst speculated that T Pyx was heading toward a dormant phase (Schaefer et al. 2010).

The 2011 outburst of T Pyx provides the community an opportunity for modern, multi-wavelength and time-series observations of a nova event and will hopefully constrain the nature of this complex system. Among these observations, X-ray grating spectra supply information on the highest ionization states of ejected material and the best constraints on the post-outburst WD photosphere.

From the day of optical discovery, 2011 April 14.29 (Waagan et al. 2011), T Pyx was monitored with the Swift telescope (Gehrels et al. 2004). Figure 1 displays the Swift $\mathrm{X}$-Ray Telescope (XRT) count rate (top) and AAVSO $V$-band magnitude (bottom) light curves. We have plotted the entire 0.3-10 keV range of the Swift light curve (Figure 1, top), but 
Table 1

T Pyxidis X-Ray and Optical Observations

\begin{tabular}{|c|c|c|c|c|c|}
\hline Date & Observatory & Instrument & $\begin{array}{l}\text { Exp. Time } \\
\quad(\mathrm{ks})\end{array}$ & $\begin{array}{l}\text { Days after } \\
\text { Outburst }\end{array}$ & $\begin{array}{c}\text { Mean Count Rate (counts ks }{ }^{-1} \text { ) } \\
0.25-1.24 \mathrm{keV}\end{array}$ \\
\hline 2011 Nov 3 & Chandra & HRC-S/LETG & 40.13 & 210 & $89.2 \pm 4.1$ \\
\hline \multirow[t]{2}{*}{2011 Nov 28} & XMM-Newton & RGS 1-2 & 30.92 & 235 & $44.5 \pm 1.1$ \\
\hline & & EPIC-pn & & & $1099 \pm 8$ \\
\hline 2012 Nov 22 & SALT & RSS PG900 & $\ldots$ & 588 & $\ldots$ \\
\hline 2012 Dec 6 & & RSS PG 2300 & $\ldots$ & 602 & $\ldots$ \\
\hline 2012 Dec 17 & & RSS PG900 & $\ldots$ & 617 & $\ldots$ \\
\hline
\end{tabular}

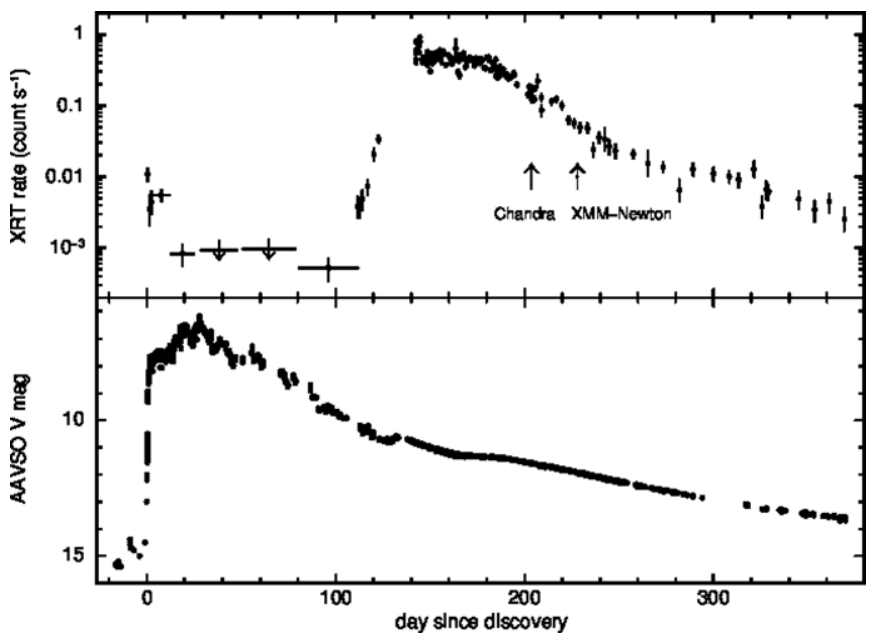

Figure 1. Top: the Swift $0.3-10 \mathrm{keV}$ XRT PC mode grade 0-12 light curve of T Pyx, shown with one bin per snapshot after $\sim 100$ days; at earlier times, some observations were combined to obtain detections or upper limits. The upper limits are calculated at $3 \sigma$ confidence according to the Bayesian method of Kraft et al. (1991). Arrows mark the dates of Chandra and XMM observations. Bottom: $V$-band CCD photometry from the AAVSO (http://www.aavso.org); only self-consistent data sets are shown.

the peak flux is dominated by soft emission in the $0.3-0.8 \mathrm{keV}$ range. A faint X-ray source was observed at the position of T Pyx (Kuulkers et al. 2011) from optical discovery to 2011 July 31 (day 105), at which point the source was no longer detectable (Osborne et al. 2011). 2011 August 3 (day 111) saw the X-ray flux begin to rise, peaking on 2011 September 6 (day 145) and plateauing for around 40 days before declining monotonically. The substantial delay in the X-ray peak is conventionally attributed to the ejection of optically thick material in the early phase of the outburst (Kato \& Hachisu 1994). As the ejected shell expands, it becomes optically thin to X-ray emission revealing the underlying WD. Because the morphology and timescale of outburst light curves are extremely variable from one RN to the next, the publicly available Swift data provide a fundamental tool for scheduling X-ray observations.

T Pyx had been detected with Swift since 2011 August, but due to a number of different planning and scheduling constraints, $\mathrm{X}$-ray grating observations were not scheduled till the end of 2011 October, when the average Swift XRT count rate had decreased from a peak of $\sim 0.6$ counts $\mathrm{s}^{-1}$ to $\sim 0.1$ counts $\mathrm{s}^{-1}$ (Chandra, 210 days) and to $\sim 0.02$ counts s $^{-1}$ (XMM, 235 days; see Figure 1).

Although the XRT plateau suggested a high luminosity, the broadband data were not ideal for the identification of the origin of the X-ray emission, whether super-soft (SSS) X-ray emission from the WD surface or unresolved emission lines in the ejected shell. This highlights the significance of obtaining X-ray grating observations during the SSS phase. Rauch et al. (2010) ${ }^{6}$ calculated a set of publicly available WD atmospheric models that place constraints on the mass, temperature, and composition of the WD. These parameters are extremely valuable in order to evaluate whether RNe are viable Type Ia supernova progenitor candidates. Having both an estimate of the WD mass and spectral features of the circumstellar material is important to trace a possible pre-supernova Ia evolution.

In this work we present the two X-ray grating observations, obtained with Chandra and XMM-Newton. We also present the first in a series of optical spectra taken with the Southern African Large Telescope (SALT) during the nova's return to quiescence. Observations and data reduction methods are presented in Section 2. Section 3 presents the analysis of the spectral and temporal components of the X-ray and optical data. Section 4 provides a discussion of our results, with a conclusion in Section 5 .

\section{DATA AND REDUCTION METHODS}

In the framework of our pre-approved guest target of opportunity program, T Pyx was observed with Chandra and XMMNewton 210 and 235 days after the 2011 April 14 discovery, respectively. Table 1 provides a summary of the X-ray and optical observations. With each satellite we utilized dispersion gratings to obtain high spectral resolution data. Optical spectra of T Pyx were also obtained with the SALT telescope in 2012 November and December.

\subsection{Chandra}

On 2011 November 3 (day 210) we obtained a 40.13 ks Chandra observation of T Pyx using the Low-Energy Transmission Grating (LETG) with the High Resolution Camera in spectroscopy mode (HRC-S). The +1 and -1 spectral orders were reduced, extracted, and combined using CIAO (version 4.2).

Even with a modest count rate, the spectrum is rich in emission lines and continuum present from $\sim 25-40 \AA$ (see Figure 2). With a spectral resolution of $R \sim 1000$ (Brinkman et al. 1997), we are able to resolve a complex line spectrum with velocity broadening on the order of $2500 \mathrm{~km} \mathrm{~s}^{-1}$ (see Section 3.1), significantly larger than the instrumental broadening.

The X-ray light curve was also extracted from the HRC with CIAO (version 4.2) and shows a periodic (factor of $\sim 2$ ) variability in the count rate across the $\sim 40 \mathrm{ks}$ observation (see Section 3.3).

\footnotetext{
6 http://astro.uni-tuebingen.de/ rauch/
} 

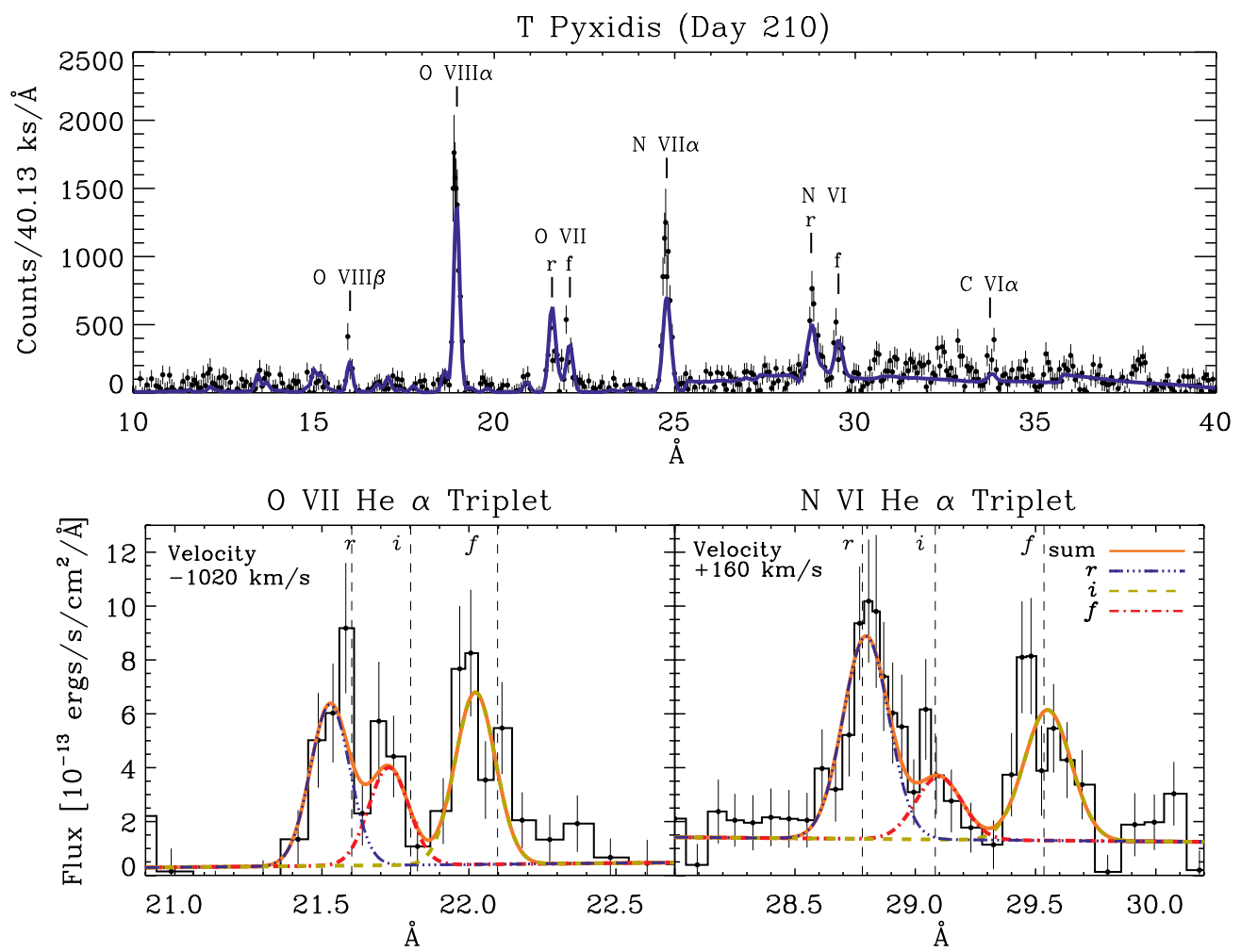

Figure 2. Top: binned Chandra spectrum of T Pyx 210 days after outburst. XSPEC best-fit model is overplotted in blue (see Table 3 for model parameters). Bottom: flux-corrected $\mathrm{O}$ VII (left) and $\mathrm{N}$ vi triplets (right) with three Gaussian fits. Vertical dashed lines represent the rest wavelengths of the $r, i$, and $f$ lines from left to right, respectively, for each triplet.

(A color version of this figure is available in the online journal.)

Near-simultaneous Swift observations yield an XRT count rate of $0.11 \pm 0.01$ and a uvm2 magnitude of $12.23 \pm 0.02$. The $V$-band magnitude at this time was $11.71 \pm 0.02$ (AAVSO).

\subsection{XMM-Newton}

The nova was observed again 235 days after outburst with XMM-Newton on 2012 November 28 for 30.92 ks. The observation was conducted with all six XMM-Newton instruments, namely, the optical monitor (OM), EPIC-pn, the two EPIC MOS (all in imaging mode), and the two RGS gratings. The pn was used in the "prime small window" mode; the MOS 1 and 2 were used in "prime full window" and "prime partial W3" modes, respectively. The thin filter was used for both MOS and pn.

The data were reduced with the XMM-Newton standard analysis system, XMM-SAS, version 11.0.2. The grating spectra show the same basic spectral properties as the Chandra spectrum of 25 days earlier only with a decrease in average flux by more than a factor 2. The EPIC-pn camera provided the highest signalto-noise ratio $(\mathrm{S} / \mathrm{N})$ light curve of our observations and showed the same variability seen in the previous Chandra observations but with lower amplitude (see Section 3.3). The OM data were taken in imaging mode with the uvw2 filter with 25 exposures lasting 800-900 s during the $\sim 30 \mathrm{ks}$ exposure. An average uvw2 magnitude of 12.35 was obtained with no clear trend or periodicity.

Adjacent Swift observations measured uvm2 magnitudes of $12.85 \pm 0.02$ and $12.71 \pm 0.02$. The count rate of the nearest XRT observation had dropped significantly from the Chandra observation to $0.024 \pm 0.005$. The AAVSO $V$-band magnitude also fell to $12.06 \pm 0.01$.

\subsection{SALT}

The SALT telescope located in Sutherland, South Africa, with $\mathrm{a} \sim 10.5 \mathrm{~m}$ diameter mirror is at the moment the largest singleprimary mirror telescope (Buckley et al. 2006). SALT spectra, the first in a planned regular monitoring in the first three years after the outburst, have already been obtained. Observations utilized the Robert Stobie Spectrograph (RSS; Burgh et al. 2003; Kobulnicky et al. 2003) in long-slit mode and with the PG 2300 and PG 900 gratings. Our low-resolution observations were made on 2012 November 22 (day 588) and 2012 December 17 (day 613) covering a 3150-6300 $\AA$ range at a spectral resolution $R \sim 800$. A higher resolution spectrum $(R \sim 3000)$ in the 4600-5100 $\AA$ wavelength range was obtained on 2012 December 6 (day 602). For information on SALT, the RSS, and its instruments see Buckley et al. (2008). The data were reduced and analyzed with the Pyraf SALT reduction pipeline (Crawford et al. 2010) and $\operatorname{IRAF}^{7}$ software packages.

\section{ANALYSIS}

\subsection{Day 210 X-Ray Spectrum}

The top panel of Figure 2 displays the binned Chandra spectrum 210 days after the outburst discovery with an XSPEC best-fit model in blue. Expanded regions around the O VII and $\mathrm{N}$ vi helium-like triplets are presented in the bottom panels. The spectrum is dominated by broad emission lines of $\mathrm{H}$ - and Helike ionization states of $\mathrm{C}, \mathrm{N}$, and $\mathrm{O}$ with low-level continuum from $25-40 \AA$.

\footnotetext{
7 IRAF is distributed by the National Optical Astronomy Observatory, which is operated by the Association of Universities for Research in Astronomy (AURA) under cooperative agreement with the National Science Foundation.
} 
Table 2

X-Ray Emission Lines

\begin{tabular}{|c|c|c|c|c|c|c|c|}
\hline \multirow[t]{2}{*}{ Ion } & \multirow[t]{2}{*}{$\begin{array}{c}\lambda_{\text {rest }} \\
(\AA)\end{array}$} & $\begin{array}{l}\lambda_{\text {obs }} \\
(\AA)\end{array}$ & $\begin{array}{l}\text { Vel. Centroid } \\
\quad\left(\mathrm{km} \mathrm{s}^{-1}\right)\end{array}$ & $\begin{array}{l}\text { FWHM } \\
\left(\mathrm{km} \mathrm{s}^{-1}\right)\end{array}$ & $\begin{array}{c}\lambda_{\mathrm{obs}} \\
(\AA)\end{array}$ & $\begin{array}{l}\text { Vel. Centroid } \\
\quad\left(\mathrm{km} \mathrm{s}^{-1}\right)\end{array}$ & $\begin{array}{l}\text { FWHM } \\
\left(\mathrm{km} \mathrm{s}^{-1}\right)\end{array}$ \\
\hline & & \multicolumn{3}{|c|}{ Day 210} & \multicolumn{3}{|c|}{ Day 235} \\
\hline $\operatorname{Ne} \mathrm{x} \operatorname{Ly} \alpha$ & 12.13 & $\ldots$ & $\ldots$ & $\ldots$ & $\ldots$ & $\ldots$ & $\ldots$ \\
\hline O vIII Ly $\beta$ & 16.01 & $15.95 \pm 0.02$ & $-1080 \pm 320$ & $1990 \pm 710$ & $\ldots$ & $\ldots$ & $\ldots$ \\
\hline O VIII Ly $\alpha$ & 18.97 & $18.94 \pm 0.01$ & $-420 \pm 100$ & $2980 \pm 220$ & $19.81 \pm 0.01$ & $-1000 \pm 90$ & $3300 \pm 200$ \\
\hline N VII Ly $\beta$ & 20.91 & $\ldots$ & $\ldots$ & $\ldots$ & $\ldots$ & $\ldots$ & $\ldots$ \\
\hline$r$ & 21.60 & $21.53 \pm 0.01$ & & & $\ldots$ & & \\
\hline $\mathrm{O}$ vII He $\alpha i$ & 21.80 & $21.73 \pm 0.01$ & $-1020 \pm 180$ & $2190 \pm 300$ & $\ldots$ & $\ldots$ & $\ldots$ \\
\hline$f$ & 22.10 & $22.02 \pm 0.01$ & & & $\cdots$ & & \\
\hline N vII Ly $\alpha$ & 24.78 & $24.77 \pm 0.01$ & $-190 \pm 110$ & $3010 \pm 260$ & $24.73 \pm 0.01$ & $-580 \pm 80$ & $2850 \pm 160$ \\
\hline$r$ & 28.78 & $28.80 \pm 0.01$ & & & $\ldots$ & & \\
\hline N VI He $\alpha i$ & 29.08 & $29.10 \pm 0.01$ & $+160 \pm 140$ & $2330 \pm 300$ & $\ldots$ & $\ldots$ & $\ldots$ \\
\hline$f$ & 29.53 & $29.55 \pm 0.01$ & & & $\ldots$ & & \\
\hline $\mathrm{C}$ vi Ly $\alpha$ & 33.73 & $\ldots$ & $\ldots$ & $\ldots$ & $\ldots$ & $\ldots$ & $\ldots$ \\
\hline
\end{tabular}

\subsubsection{XSPEC Spectral Modeling Procedure}

Emission-line and continuum model fitting is done simultaneously in XSPEC using $\chi^{2}$ statistics to determine the goodness of fit with the Churazov weighting scheme (Churazov et al. 1996), commonly used in low-count spectral fitting. Models are fit to unbinned data but are presented in a binned format for clarity. For consistency we also fit the data using the $C$-statistic method (Cash 1979) at various binnings and find equivalent results. The final model is presented as the overplotted blue line in the top panel of Figure 2. All components were fit with a single $N(\mathrm{H})$ value that agrees with reddening estimates and the expected column density to T Pyx (Shore et al. 2013).

\subsubsection{Emission Lines}

Table 2 presents the line center, velocity centroid, and FWHM for the most prominent X-ray emission lines. Detected emission lines with lower $\mathrm{S} / \mathrm{N}$ are only listed as being present, but no attempt was made to characterize them. These estimates were made by fitting a Gaussian profile to each flux-corrected line profile. Fits were made to data binned to a minimum of 20 counts per bin to justify $\chi^{2}$ minimization and increase $\mathrm{S} / \mathrm{N}$. As a result, the bin size presented in Figure 2 (bottom) is variable with a mean width of $\sim 0.1 \AA$. Flux conversion from instrumental counts was done using the ISIS software package flux_corr. This preforms a proper division of data by the auxiliary response file while accounting for the line shape by also integrating over the redistribution matrix file. ISIS flux conversion can have an appreciable uncertainty in absolute flux over a broad wavelength range; however, the narrow He-like triplet ranges $(\sim 2 \AA)$ over which we are concerned with relative flux ratios are robust (see below). We note that while the intrinsic line shape may not be Gaussian in nature, at the resolution of our data it provides a good first-order representation.

The He-like triplets of oxygen and nitrogen show blending between the resonance $(r)$, intercombination $(i)$, and forbidden $(f)$ lines (see Figure 2 bottom panel, labeled vertical lines). We fit the blends simultaneously with three Gaussians requiring a constant FWHM and velocity shift across the triplet. We justify this constraint as it is expected that the emitting material is under similar physical conditions. This provides reasonable agreement with centroid shifts and line widths of unblended $\mathrm{H}$-like lines.
Data for He-like triplets in Table 2 are presented as the best-fit velocity centroid and FWHM of the three lines.

Blends from other emission lines may be present in the data contaminating our estimates from Gaussian fitting such as Fe XVIII $(16.20 \AA)$ with O VIII Ly $\beta$ (16.01) and the N VI He $\beta$ resonance line $(\sim 24.89 \AA)$ with N vil Ly $\alpha$. The former we conclude to be negligible due to the absence of other $\mathrm{Fe}$ emission lines in the spectrum. The latter is likely to have some level of contamination, but given the plasma temperature we derive from the $\mathrm{N}$ vII He-like triplet (see below), the expected contribution of $\mathrm{N}$ VI He $\beta$ (from an APEC model) is meager compared to N viI Ly $\alpha$.

In order to describe the ionization and excitation mechanism behind the observed emission lines, as well as placing estimates on the electron temperature and density of the emitting material, we analyze the He-like triplet flux ratios of $\mathrm{N}$ VI and O VII using diagnostics from Porquet et al. (2001) and Blumenthal et al. (1972). We calculate the $G$ ratio, $(f+i) / r$, which is sensitive to the ionization mechanism and electron temperature, where $G$ ratios $\sim 1$ indicate a plasma in collisional ionization equilibrium (CIE). Larger values indicate contributions of photoionization (Porquet \& Dubau 2000). We also utilize the $R$ ratio, $f / i$, which is sensitive to electron density. Triplet diagnostics require energy flux ratios, not instrumental counts, hence the need for ISIS flux conversion. From the N vi triplet, we calculate a $G$ ratio of $G_{N}=1.0 \pm 0.2$, which is consistent with a plasma in CIE at $T_{e}=1.1_{-0.2}^{+0.2} \times 10^{6} \mathrm{~K}$.

The $R$ ratio yields $R_{N}=2.1 \pm 0.8$, which corresponds to a density of $n_{e}=(8 \pm 6) \times 10^{9} \mathrm{~cm}^{-3}$ assuming only collisional excitation. The $R(f / i)$ diagnostic can be strongly influenced by UV radiation, which excites $f$ level electrons into the $i$ level, decreasing the $R$ ratio and suggesting erroneously large densities. This effect is extreme in cases where the emitting material is near a strong UV emitter (e.g., O star winds where $R$ is typically $\sim 1$ ). In the case of T Pyx, we conclude that our result is largely unaffected by UV radiation, given that: (1) the UV flux measured with Swift is low at this stage of the outburst, (2) the emitting material is in the ejecta and likely far from the WD (at a maximum outflow velocity of $\sim 4000 \mathrm{~km} \mathrm{~s}^{-1}$ found by Shore et al. 2011, a distance $>10^{10} \mathrm{~km}$ is found for a constant velocity outflow (Shore et al. 2013) at day 210), and (3) the $R$ value obtained falls in the steepest portion of the $R\left(n_{e}\right)$ curve where electron density is relatively insensitive to $R$ (see Porquet \& Dubau 2000; Figure 8). With only loose constraints on the UV 
Table 3

Physical Parameters of the Fits Shown in Figures 2, 3, and 4

\begin{tabular}{|c|c|c|c|}
\hline \multirow[t]{2}{*}{ Parameter $^{\mathrm{a}}$} & \multirow[t]{2}{*}{$\begin{array}{c}2011 \text { Nov } 3 \\
\text { Day } 210\end{array}$} & \multicolumn{2}{|c|}{$\begin{array}{l}2011 \text { Nov } 28 \\
\text { Day } 235\end{array}$} \\
\hline & & Fit $N(\mathrm{H})$ & Fixed $N(\mathrm{H})$ \\
\hline $\begin{array}{l}N(\mathrm{H})\left(10^{21} \mathrm{~cm}^{-2}\right) \\
\mathrm{N} / \mathrm{H}^{\mathrm{b}} \\
F_{\text {Model }}\left(10^{-12} \mathrm{erg} \mathrm{cm}^{-2} \mathrm{~s}^{-1}\right)\end{array}$ & $\begin{array}{c}1.5(0.1) \\
15 \\
4.08\end{array}$ & $\begin{array}{c}1.6(0.1) \\
15 \\
1.81\end{array}$ & $\begin{array}{c}1.5 \\
15 \\
2.04 \\
\end{array}$ \\
\hline $\begin{array}{l}T_{\mathrm{WD}}(\mathrm{K}) \\
R_{\mathrm{WD}} \times(d / 4.8 \mathrm{kpc})(\mathrm{km}) \\
F_{\mathrm{WD}}\left(10^{-12} \mathrm{erg} \mathrm{cm}^{-2} \mathrm{~s}^{-1}\right)^{\mathrm{c}} \\
F_{\mathrm{WD}, \text { un }}\left(10^{-12} \mathrm{erg} \mathrm{cm}^{-2} \mathrm{~s}^{-1}\right)^{\mathrm{d}} \\
\end{array}$ & $\begin{array}{c}417,000(22,500) \\
3,050(1,670) \\
2.15 \\
53.8 \\
\end{array}$ & $\begin{array}{c}405,000(22,500) \\
3,050 \\
0.61 \\
40.61 \\
\end{array}$ & $\begin{array}{c}404,000(22,500) \\
3,050 \\
0.90 \\
40.34 \\
\end{array}$ \\
\hline $\begin{array}{l}k T_{\mathrm{P}, 1}(\mathrm{eV}) \\
\text { Vol. em. measure } \times(d / 4.8 \mathrm{kpc})^{2}\left(10^{55} \mathrm{~cm}^{-3}\right) \\
\mathrm{v}\left(\mathrm{FWHM}, \mathrm{km} \mathrm{s}^{-1}\right) \\
\text { Centroid shift }\left(\mathrm{km} \mathrm{s}^{-1}\right)\end{array}$ & $\begin{array}{c}94(13) \\
51(43) \\
2,380(390) \\
90(570)\end{array}$ & $\begin{array}{c}116(3) \\
36(6) \\
2,360(250) \\
-30(1,360) \\
\end{array}$ & $\begin{array}{c}115(3) \\
30(4) \\
2,350(250) \\
-310(1510) \\
\end{array}$ \\
\hline $\begin{array}{l}k T_{\mathrm{P}, 2}(\mathrm{eV}) \\
\text { Vol. em. measure } \times(d / 4.8 \mathrm{kpc})^{2}\left(10^{55} \mathrm{~cm}^{-3}\right) \\
\mathrm{v}\left(\mathrm{FWHM}, \mathrm{km} \mathrm{s}^{-1}\right) \\
\text { Centroid shift }\left(\mathrm{km} \mathrm{s}^{-1}\right)\end{array}$ & $\begin{array}{c}240(10) \\
28(8) \\
2,870(180) \\
-150(2,580)\end{array}$ & $\begin{array}{c}250(10) \\
14(1) \\
2,690(190) \\
-610(1,870)\end{array}$ & $\begin{array}{c}250(10) \\
12(1) \\
2,680(190) \\
-990(2,080)\end{array}$ \\
\hline $\begin{array}{l}k T_{\mathrm{P}, 3}(\mathrm{eV}) \\
\text { Vol. em. measure } \times(d / 4.8 \mathrm{kpc})^{2}\left(10^{55} \mathrm{~cm}^{-3}\right)\end{array}$ & & $\begin{array}{c}1,260(30) \\
6(1)\end{array}$ & $\begin{array}{l}1,250(40) \\
5(1)\end{array}$ \\
\hline
\end{tabular}

Notes.

${ }^{\text {a }}$ Component order: Tuebingen-Boulder ISM absorption model, Rauch WD atmosphere, BVAPEC 1, BVAPEC 2, BVAPEC 3. $1 \sigma$ confidence levels are given in parentheses.

${ }^{\mathrm{b}} \mathrm{N} / \mathrm{H}$ indicates nitrogen mass fraction compared to solar.

${ }^{\mathrm{c}}$ Fluxes are measured in the $0.25-1.24 \mathrm{keV}$ range.

d The "un" subscript refers to the "unabsorbed" flux.

flux and the distance of the emitting material, this density should be considered an upper limit. Results are, however, consistent with values derived for other novae (e.g., U Sco, Orio et al. 2013; Nova Cygni 1975, Neff et al. 1978).

Applying the same method to the $\mathrm{O}$ viI triplet, we find a $G$ ratio of $G_{o}=1.7 \pm 0.5$ and an $R$ ratio of $R_{o}=1.8 \pm 0.7$. These estimates provide an electron temperature of $T_{e}=$ $0.5_{-0.2}^{+0.5} \times 10^{6} \mathrm{~K}$ and a density of $n_{e}=(3 \pm 3) \times 10^{10} \mathrm{~cm}^{-3}$, again assuming that the plasma is in CIE with no contamination of UV flux. While these values agree with those from the N VI triplet, we note the lower temperature derived from $\mathrm{O}$ VII. In contrast, the O VIII $\operatorname{Ly} \beta(16.01 \AA)$ to $\operatorname{Ly} \alpha(18.97 \AA)$ ratio gives a temperature of $T_{e}>2.5 \times 10^{6} \mathrm{~K}$ (assuming CIE; Smith et al. 2001).

The discrepancy of the $\mathrm{O}$ vIII $\mathrm{Ly} \beta / \alpha$ and $\mathrm{O}$ vII triplet electron temperature diagnostics may be due to many factors that can influence the relative flux ratios of He-like triplets. Firstly, we note that the $\mathrm{O}$ vII $G$ ratio is elevated for a purely collisionally ionized plasma (usually $\sim 1$ ). One possibility, if the ratio is unaltered (by scattering for instance), is the presence of partial photoionization. This seems unlikely, however, given the minimal continuum flux in the region. Alternatively, geometrydependent resonance scattering can also effectively increase or reduce the $r$ line affecting the $G$ ratio (Porquet et al. 2010). Modeling the scattering plasma geometry is beyond the scope of this paper, and neither effect can be definitively ruled out.

Given the above discussion, we adopt the $\mathrm{O}$ VIII $\operatorname{Ly} \beta / \alpha$ ratio and $\mathrm{N}$ vi triplet temperature diagnostics assuming a plasma in CIE with multiple temperature components. The N vi triplet diagnostic is more robust than O VII because, even near the continuum emission, it shows no signature of photoionization, and if this system is at all similar to the 2010 outburst of RN U Sco, softer lines are emitted further from the WD than harder lines, decreasing the possibility of scattering (see Ness et al. 2012). WD continuum models from Rauch et al. (2010) also predict negligible atmospheric absorption at these continuum levels compared to the observed line flux. Requiring multiple plasma temperature components is not unexpected given the spatial complexity of the ejecta (Shore et al. 2013) and broad range of ionization potentials observed.

Informed by our flux-corrected triplet diagnostics, we apply an APEC model (Smith et al. 2001) in XSPEC at the N vi temperature $\left(10^{6} \mathrm{~K}\right)$ for the initial model. The APEC model assumes optically thin plasma in CIE. To account for the non-solar abundances of nova ejecta and in order to fit the velocity broadening of the lines, we chose the APEC model that includes velocity broadening and ad hoc abundances of different elements (BVAPEC). We find a good fit to the softer lines redward of $25 \AA$ with an increased nitrogen abundance $(\sim 15$ times the solar $\mathrm{N} / \mathrm{H}$ mass ratio). A high $\mathrm{N}$ abundance is expected for $\mathrm{CNO}-$ cycle processed material, so this rough estimate seems a reasonable value for modeling purposes.

The best-fit model has a velocity-broadened FWHM of $2400 \pm 200 \mathrm{~km} \mathrm{~s}^{-1}$ (similar to the low-energy lines of Table 2) and a centroid shift consistent with zero. This single model component, however, greatly underestimates the emission lines blueward of $25 \AA$ and thus confirms the need for two plasma components.

As a second component in the fit, we add an additional variable-abundance, velocity-broadened, BVAPEC model with the $\mathrm{O}$ VIII $\mathrm{Ly} \beta / \alpha$ plasma temperature $\left(2.5 \times 10^{6} \mathrm{~K}\right)$ and find a good fit to the harder emission lines with the same enhanced nitrogen abundance and velocity broadening of $2600 \pm 200 \mathrm{~km} \mathrm{~s}^{-1}$. This additional model component reduced $\chi^{2}$ from 1.10 to 1.07 (including a continuum model; see below). 

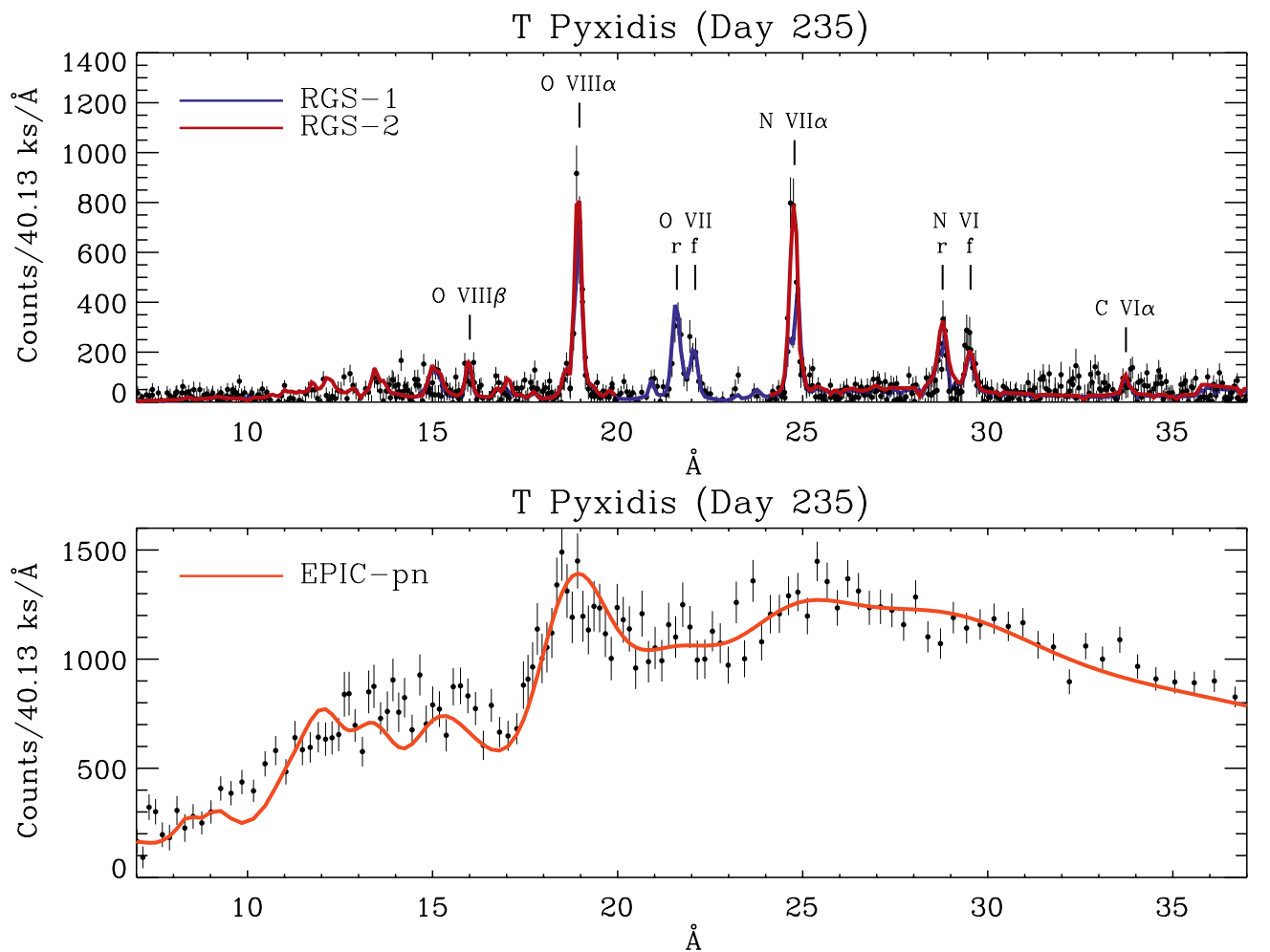

Figure 3. Binned XMM-Newton spectra 235 days after discovery. Top: RGS-1 and RGS-2 spectra with model overlaid in blue and red, respectively (model "Fit $N(\mathrm{H})$ " from Table 3). Bottom: concurrent EPIC-pn spectrum with final model overplotted. Presented model was fit simultaneously to RGS 1 and 2 and EPIC-pn data.

(A color version of this figure is available in the online journal.)

The blueshift and broadening parameters of these model components are less than but in general agreement with the trends of Table 2. The uncertainties on the velocity widths and centroid shifts are large owing to the low $\mathrm{S} / \mathrm{N}$ of the data and the attempt to fit a single set of parameters to lines of varying widths and centroid shifts (see Table 3).

\subsubsection{Continuum}

From the BVAPEC models above, the observed continuum emission is too large to be due to bremsstrahlung alone. Although an ad hoc bremsstrahlung component can also produce a higher continuum in the right range, it does not yield a good fit.

To describe the continuum, we used WD atmospheric models (Rauch et al. 2010) and found good fits to the data with reduced $\chi^{2} \simeq 1$. We fitted models using grids of $\log (g)$, with the given steps of 0.5 in $\log (g)$. We found that models with $\log (g)<8$ do not fit the data. Models with $\log (g) \geqslant 9$ reach the lower bound $(500,000 \mathrm{~K})$ of the physical temperature range before converging on a significant fit. Since the Rauch et al. (2010) models are most discrepant in the presence of strong absorption features and flux below $20 \AA$, of which we observe neither, we obtain almost equally good fits with $\log (g)=8$ and 8.5. Given that there is no significant difference in the derived temperature between these models, we present here results with $\log (g)=8$, closer to values indicated in the theoretical outburst model for T Pyx (Starrfield et al. 2012).

The atmospheric models with $\log (g)=8$ yield a best fit with $T_{\text {eff }} \sim 420,000 \mathrm{~K}$ and a $N(\mathrm{H})$ consistent with reddening estimates from Shore et al. (2013). Emission from this continuum model sharply cuts off at wavelengths short of $\sim 25 \AA$. WD models of lower temperature quickly begin to underrepresent the continuum flux in the 25-30 ̊ range. This final model with the emission-line components detailed above is presented in blue in the top panel of Figure 2. Table 3 contains full model parameters with $1 \sigma$ confidence levels in parentheses. In all parameters we have chosen a distance to T Pyx of $4.8 \mathrm{kpc}$ based on recent light echo observations with the Hubble Space Telescope Wide Field Camera 3 (Sokoloski et al. 2013).

We obtain an unabsorbed X-ray luminosity of $1.48 \times$ $10^{35} \mathrm{erg} \mathrm{s}^{-1}$ in the $0.25-1.24 \mathrm{keV}$ range. This corresponds to a bolometric luminosity of only $\sim 10^{36} \mathrm{erg} \mathrm{s}^{-1}$, while the expected luminosity for a WD at this temperature is $L_{\mathrm{Bol}} \geqslant$ $3 \times 10^{37} \mathrm{erg} \mathrm{s}^{-1}$, corresponding to a mass $\leqslant \sim 1 M_{\odot}$ (Starrfield et al. 2012). Given the lack of detected absorption features in our spectra, we can provide only an upper limit on the WD effective temperature, while $\log (g)$ and surface abundances remain only loosely constrained.

Because T Pyx is thought to be a low-inclination system, an explanation of the low luminosity in terms of a hidden WD seen through Thomson scattering coronae, like in U Sco (Ness et al. 2012; Orio et al. 2013), does not apply. The flux we measure would be consistent only with a partially obscured WD with ejecta that is still partly optically thick to the X-rays. We discuss this explanation more in Section 4.

\subsection{Day 235 X-Ray Spectra}

Although the average flux is lower by more than a factor of two in the XMM-Newton spectra, it is reduced by as much as $70 \%$ in the $30-40 \AA$ range, where the WD continuum dominates. Figure 3 displays the two RGS grating spectra on top and the EPIC-pn spectrum on bottom with best-fit models overlaid. Interestingly, the peak in the continuum and the flux from 25-30 $\AA$ is still present with the same spectral shape only at decreased levels. Qualitatively, this would require a decrease in flux while maintaining the WD temperature. 


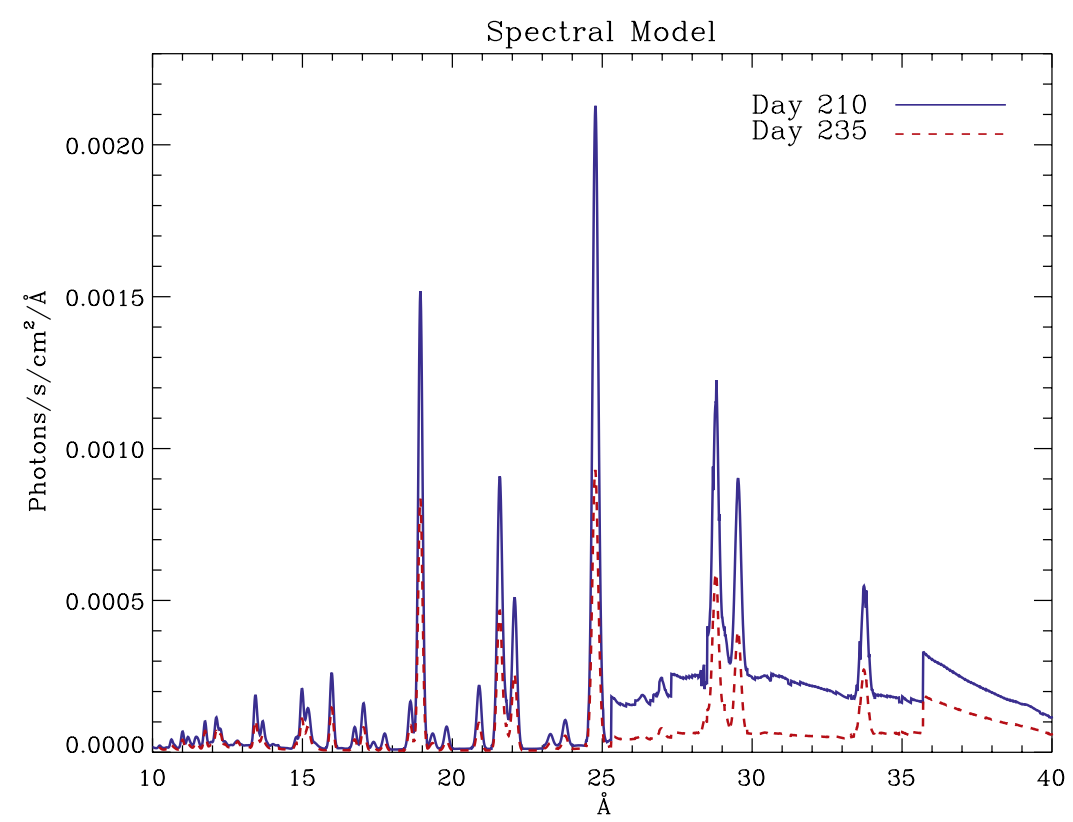

Figure 4. Evolution of the XSPEC X-ray best-fit model from 210 (blue solid) to 235 (red dotted) days after discovery. See Table 3 for best-fit parameters, day 235 is the middle, "Fit $N(\mathrm{H})$," column.

(A color version of this figure is available in the online journal.)

We simultaneously fit the RGS-1, RGS-2, and EPIC-pn spectra, shown in Figure 3, with the method described in Section 3.1.1. Implementing the best-fit parameters from day 210 as an initial model, we find that the continuum behavior can be reproduced in three ways that are degenerate to our data: (1) a $10 \%$ increase in the model $N(\mathrm{H})$ with a $3 \%$ drop in WD temperature (within $1 \sigma$ confidence limit of day 210 fit temperature) and constant WD radius (model "Fit $N(\mathrm{H})$ " in Table 3); (2) a slightly larger decrease in WD temperature (again smaller than $1 \sigma$ confidence level) while $N(\mathrm{H})$ and radius remain constant (model "Fixed $N(\mathrm{H})$ " in Table 3); and (3) a $\sim 20 \%$ decrease in the WD radius while WD temperature and $N(\mathrm{H})$ remain constant (not included; see Section 4). Insignificant statistical difference is found between these models, and all produce acceptable fits. Figure 4 shows the two best-fit models for day 210 and day 235 ("Fit $N(\mathrm{H})$ " in Table 3). The spectral shape between models varies little, and the "Fit $N(\mathrm{H})$ " model from Table 3 is presented in Figures 3 and 4.

The two plasma components show little variation in temperature but a decrease in flux from 25 days earlier. Parameters for centroid shift and velocity broadening remain uncertain as in the Chandra spectrum. Line ratios and the $G$ and $R$ quantities mentioned above appear roughly the same as 25 days earlier, but due to the reduced $\mathrm{S} / \mathrm{N}$, no attempt was made to fit them. Fits to the N VII Ly $\alpha$ and $\mathrm{O}$ VIII Ly $\alpha$ lines are presented in the three right columns of Table 2.

The inclusion of the EPIC-pn data revealed an extra source of hard emission in the 5-15 $\mathrm{A}$ range that was underrepresented by the initial model from day 210 . We find that this emission can be modeled with a third plasma component at $T_{\mathrm{e}}=\sim 15 \times 10^{6} \mathrm{~K}$ with similar plasma characteristics as the lower temperature components. The values of this model component are less certain because it is mainly constrained by the EPIC-pn broadband data. The addition of this component improved the reduced $\chi^{2}$ by 0.13 to 0.99 . We do not believe that this marks the emergence of a harder emission component, only that it went undetected in the Chandra (day 210) spectrum due to low $\mathrm{S} / \mathrm{N}$.

\subsection{X-Ray Light Curve and Periodicity}

The long-seen periodic variability in non-outburst optical photometry of T Pyx was determined to be orbital in nature by Uthas et al. (2010). These authors applied the most recent optical photometric ephemeris timing from ongoing work by Patterson et al. (2013), updated from Patterson et al. (1998), with the help of additional observations from the Center for Backyard Astronomy. Timing for the optical ephemeris has the form

$$
\begin{aligned}
\text { Minimum Light }(\mathrm{HJD})= & 2,451,651.65255(35) \\
& +0.076227249(16) \times N \\
& +2.546(54) \times 10^{-11} N^{2},
\end{aligned}
$$

where $N$ is the number of periods since the given epoch. Numbers in parentheses are the error of the last two significant digits (Uthas et al. 2010). The $N^{2}$ term is for a lengthening period, believed to be due to a high mass accretion rate (Patterson et al. 1998), and was required for agreement with the spectroscopic period.

We compute a Lomb-Scargle periodogram (Scargle 1982) of the heliocentric corrected EPIC-pn, MOS, and HRC-LETG light curves. The top left panel of Figure 5 displays the EPIC-pn periodogram. The main peak is well above the $1 \%$ false-alarm probability (horizontal dashed line). The vertical dashed line represents the extrapolated optical frequency for our epoch of observation from Equation (1). While only a small frequency window is displayed, no other frequencies produced statistically significant power.

To estimate the error in our peak frequency, we perform a Monte Carlo bootstrap error simulation of 100,000 iterations. The resultant probability distribution function is presented in the top right panel of Figure 5. The solid vertical line displays the periodogram peak (from the left panel; Figure 5), and the dashed line, again, represents the extrapolated frequency from Equation (1). The hatched region marks the $68.3 \%(1 \sigma)$ enclosure of the cumulative distribution function 

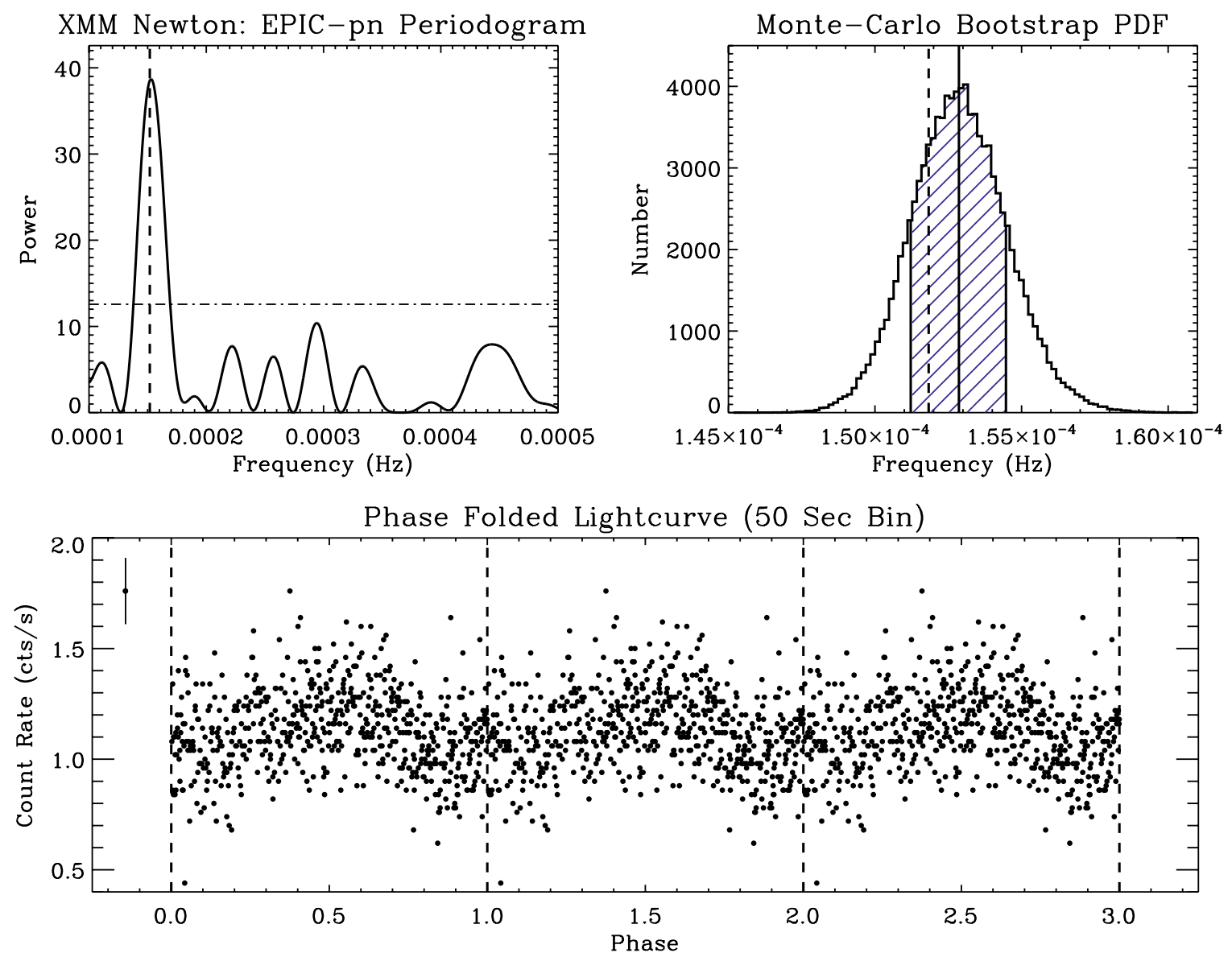

Figure 5. Top left: Lomb-Scargle periodogram of the XMM-Newton, EPIC-pn light curve (235 days after outburst). Dot-dashed horizontal line displays the 1\% false-alarm probability level. Dashed vertical line represents the extrapolated optical period for the epoch of observation (1.830 hr). Top right: probability distribution function of Monte Carlo bootstrap error simulation. Solid vertical line represents periodogram peak from left panel (1.817 $\pm 0.019 \mathrm{hr})$. Dashed vertical line again represents expected optical period. The shaded region encloses $68.3 \%$ of the error distribution. Bottom: phase folded light curve of data binned at $50 \mathrm{~s}$. Leftmost point displays the average error bar; phase zero is set to the optical ephemeris time. Data are repeated over three phases to highlight modulation pattern.

(A color version of this figure is available in the online journal.)

(not shown). Our result is an X-ray period of $1.817 \pm 0.019 \mathrm{hr}$ $(0.07571 \pm 0.00081$ days $)$, which is consistent with the predicted optical period for this epoch, $1.830 \mathrm{hr}$. While we only present the full EPIC-pn timing analysis herein for brevity, the same analysis was carried out for the MOS and HRC-LETG light curves. Both independently recovered the optical period of the above analysis but with larger uncertainties owing to their lower sensitivities. We note that prior to the outburst Balman (2012) recovered the optical period in X-rays during quiescence using the Chandra ACIS-S detector.

Recovering the same period at X-ray and optical wavelengths places constraints on the physical process behind the variability. The bottom panel of Figure 5 presents the EPIC-pn phase-folded light curve (day 235) in $50 \mathrm{~s}$ bins. The leftmost point displays the average error bar. The optical ephemeris time is set to phase zero (plotted as vertical dashed lines), where there is a clear match to the $\mathrm{X}$-ray minimum.

In the top panel of Figure 6 the Chandra HRC (day 210) light curve is presented with the optical ephemerides over-plotted in dashed vertical lines. The bottom panel displays the phasefolded light curve about its independently measured, consistent period. The noise is much higher compared to the EPIC-pn but shows the same match to the optical ephemeris. We note that individual periods show significant variability that can be seen in the sharp rise at $\sim 0.57$ and $\sim 0.69 \operatorname{HJD}(-2,455,869)$ of the HRC light curve.

To investigate the spectral region of variability, we extract the Chandra spectrum (highest $\mathrm{S} / \mathrm{N}$ ) at elevated (0.4-0.7) and minimum (0.85-1.15) phases and present the differenced result in Figure 7. The variability occurs predominantly redward of the $\mathrm{N}$ vi triplet emission lines and is primarily in the continuum flux. In Section 4 we comment on possible sources of the periodic variation originating either from occultation on the suggested orbital period or instead due to the WD spin.

\subsection{SALT Optical Spectra}

Two groups found that the mass ejected in the $2011 \mathrm{~T}$ Pyx outburst was "bipolar" in nature, Chesneau et al. (2011) only a few days and six weeks after the outburst, and Shore et al. (2013) six months and one year post-outburst. This structure, if it persisted in November of 2011, may well explain a partial obscuration of the WD surface inferred from our $\mathrm{X}$-ray grating spectra. In order to assess whether the ejecta expansion continued without spherical symmetry and to obtain further insight into the geometry and evolution of the shell, we obtained additional optical spectra at a later epoch. We observed the nova with SALT and the PG 2300 grating $(R \sim 3000)$ in the 4480-5516 $\AA$ region on 2012 December 6, and at 

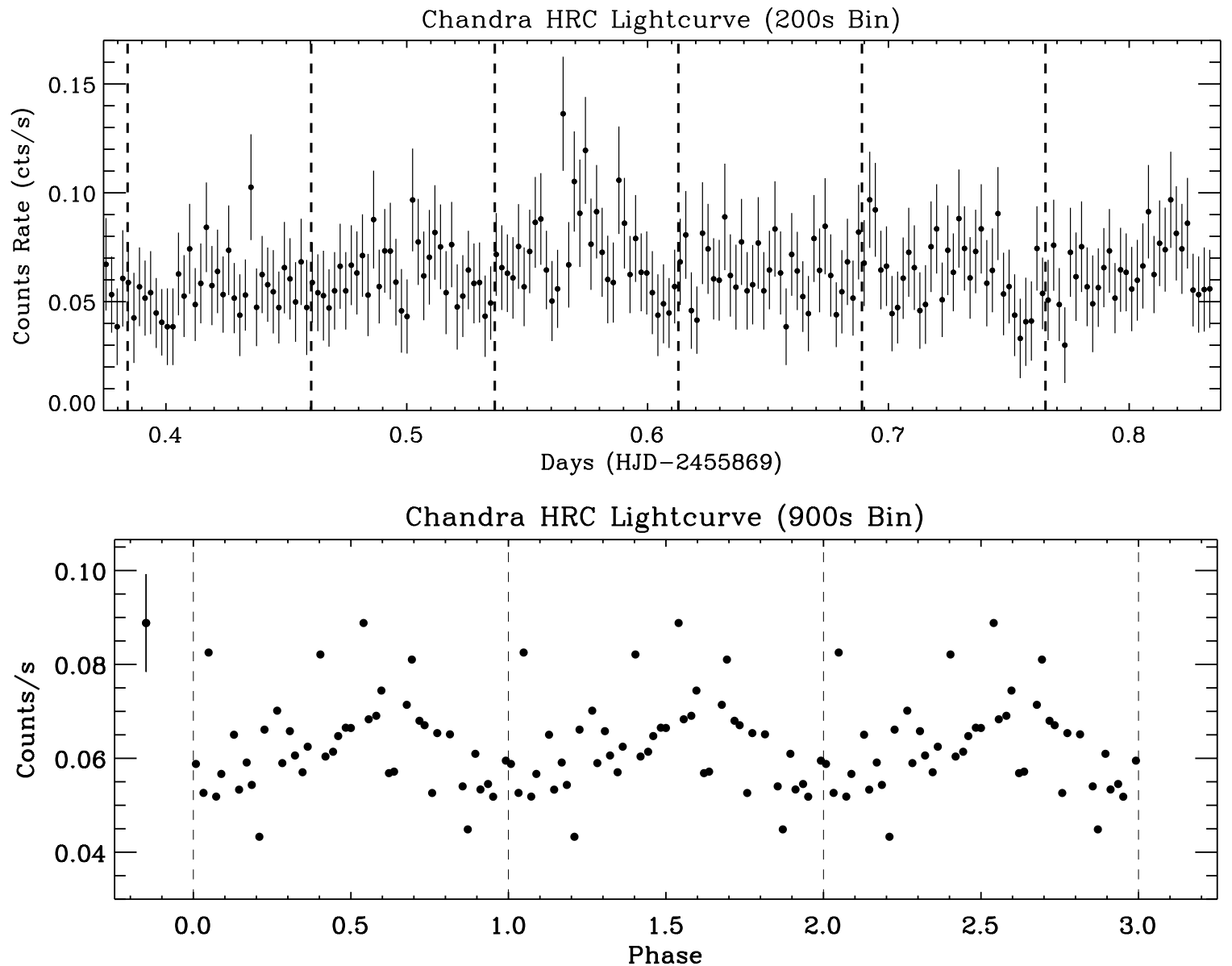

Figure 6. Top: Chandra-HRC light curve 210 days after outburst. Vertical dashed lines are predicted optical ephemerides from Equation (1). Bottom: phase-folded light curve of independently found period (agrees with extrapolated optical period). Phase zero is set to the optical ephemeris time.

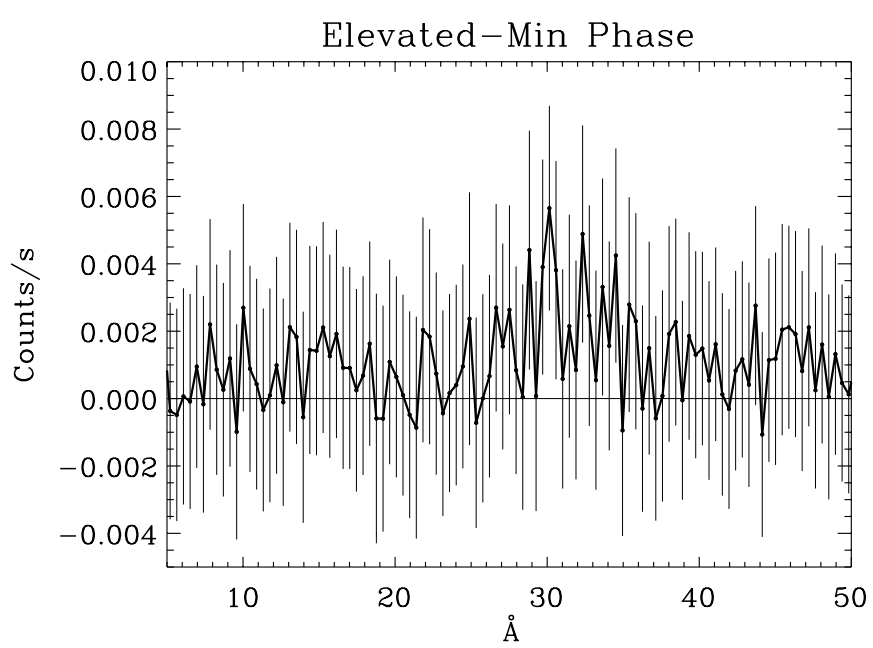

Figure 7. Difference of Chandra spectrum extracted in high and low phases. Elevated spectra include phase $0.4-0.7$; minimum spectra include phases $0.0-0.15$ and $0.85-1.0$ (compare phase intervals with Figures 5 and 6). Result is binned by a factor of 30 from native resolution.

lower resolution with the PG 900 grating $(R \sim 800)$ in the 3156-6318 $\AA$ region on 2012 November 22 and on 2012 December 17 . There was no significant change in the spectra between these dates.

We found that in December of 2012 (602 days after outburst), T Pyx was still in the nebular phase due to the strong emission of nebular forbidden lines of [O III] (Snijders 1990). Figure 8 shows a full, low-resolution spectrum from 2012 November 22 in the top panel. An enlarged region of the higher resolution spectrum from 2012 December 6 around the [O III] doublet (4959, $5007 \AA)$ and He II (4686 $\AA$ ) and C/N Bowen blend are presented on the bottom left and right, respectively. The $\mathrm{C} / \mathrm{N}$ Bowen blend (4640-4650 $\AA$ ) consists of several lines of N III and $\mathrm{C}$ III that are seen in cataclysmic variables and low-mass $\mathrm{X}$-ray binaries with high mass accretion rates and in novae during outburst. Relative flux calibration across the spectrum is presented in the bottom panel of Figure 8, but the scale is arbitrary.

We compare the equivalent width ratio of the above lines to $\mathrm{H} \beta$ with other recent novae (V382 Vel, V4743 Sgr, and KT Eri) also observed with SALT ranging from 2.5 to $15 \mathrm{yr}$ after outburst (M. Orio et al., in preparation). We find that the [O III] line intensity in T Pyx is still very large and does not decay as expected (Downes et al. 2001). This is interesting considering that in most RN, “[O III] $5007 \AA$ is usually inconspicuous or absent" (Downes et al. 2001) and generally only in "very slow novae" is the [O III] emission prominent more than a year after the outburst. The long nebular phase is thus unexpected in $\mathrm{T}$ Pyx not only because it is not as slow as most novae with longlasting nebular lines, but also because of the small amount of ejecta mass that has been estimated (e.g., Shore et al. 2013). Novae with comparable velocity and ejecta mass appear to have much shorter nebular phases. This will be discussed more in detail in an upcoming article (M. Orio et al., in preparation). 

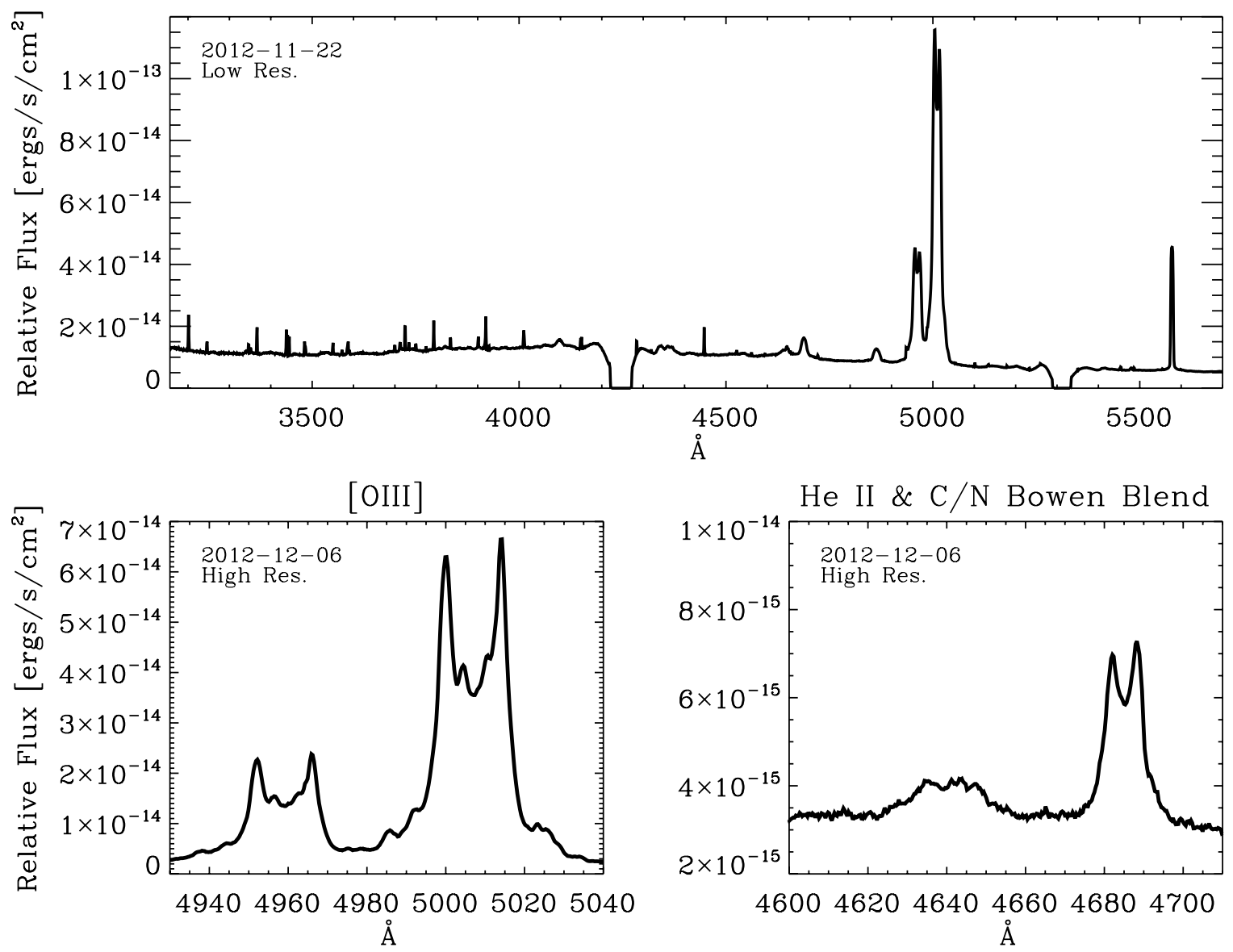

Figure 8. SALT optical spectra of the T Pyx nebular phase. Top: full low-resolution spectrum from 2012 November 22. Bottom: regions of high-resolution spectrum from 2012 December 6. [O III] lines $\lambda \lambda 4960$ and 5007 are presented in the left panel with He II $\lambda 4686$ and C/N Bowen blend (4640-4650 $\AA$ ) in the right. Flux is arbitrarily normalized but consistent across bottom panels. Wavelength scales are equivalent in bottom panels, but notice difference in flux scale and profile shape.

The ratio of the equivalent width of the He II line at $4686 \AA$ to that of $\mathrm{H} \beta$ is lower than it has been observed in novae that have been super-soft X-ray sources with effective temperatures above $500,000 \mathrm{~K}$. Moreover, in the sample of objects studied by M. Orio et al. (in preparation), the He II line at $4686 \AA$ is still more prominent than $\mathrm{H} \beta$ even $14 \mathrm{yr}$ after the outburst. The ratio of these lines depends mainly on the temperature of the ionizing source and the amount of total ionizing radiation absorbed by the nebular material (Szczerba 1987). Assuming that these lines trace the same ejected material, this measurement may imply that the super-soft X-ray source was less hot and potentially cooled more rapidly in T Pyx than in other recent novae observed as super-soft X-ray sources.

Unlike in other RNe at this post-outburst epoch, the [O III] line at $5007 \AA$ has more than 10 -fold the flux of the He II line (Downes et al. 2001). The [O III] lines are due to forbidden transitions that require a critical density of $n_{e} \leqslant 10^{5} \mathrm{~cm}^{-3}$. The nebular material is far from being spherically distributed and has a low filling factor $f$, which diagnostics from Shore et al. (2011) estimate to be $f=0.03$.

The spectral resolution of the PG 900 grating is only of about $6.5 \AA$ at the wavelength of the [O III] lines, so we will focus on the structure of the lines observed with the PG 2300 grating, which has a resolution of $1.5 \AA$ (Figure 8 , bottom). Figure 9 displays the velocity structure of the [O III] and He II lines in the left panel with an enlarged region around the He II line in the right panel. In our spectra, the line profiles still have nearly the same structure detected in 2011 October (day 180) and modeled as bipolar by Shore et al. (2013) with an inner structure apparently due to a separate bipolar ring at a different opening angle. Both observations have similar relative weights between the broader and narrower bipolar components forming the lines at this late stage (see Figure 11 of Shore et al. 2013). The blue wing of the line is more prominent than the red wing, possibly indicating a departure from the axisymmetric conical geometry.

The He II line at $4686 \AA$ is much less prominent and has a markedly different velocity structure than the [O III] lines. A double Gaussian fit to He II $\lambda 4686$ yields an FWHM of 328 and $317 \mathrm{~km} \mathrm{~s}^{-1}$ and separation of $384 \mathrm{~km} \mathrm{~s}^{-1}$. These values are comparable to the average FWHM of $450 \mathrm{~km} \mathrm{~s}^{-1}$ and separation of $300 \mathrm{~km} \mathrm{~s}^{-1}$ used by Uthas et al. (2010) to describe pre-outburst He II disk emission. It is possible that this emission is coming from a reforming accretion disk. Since the spectra we observed with SALT are very different from the pre-outburst spectra, we assume that the disk was disrupted or at least disturbed in the outburst. The $\mathrm{C} / \mathrm{N}$ Bowen blend in particular is much less prominent in our spectra, while $\mathrm{He}$ I emission features at $4921 \AA$ and $5015 \AA$ are absent. These lines were dominant in the pre-outburst spectra and were attributed to the accretion disk. Therefore, it seems unlikely that the disk has been completely reformed, although it may be in the process of reforming.

It is also possible that the He II emission is not associated with a reforming accretion disk, but instead may originate in a separate structure of the ejecta. Nelson et al. (2012) suggest 

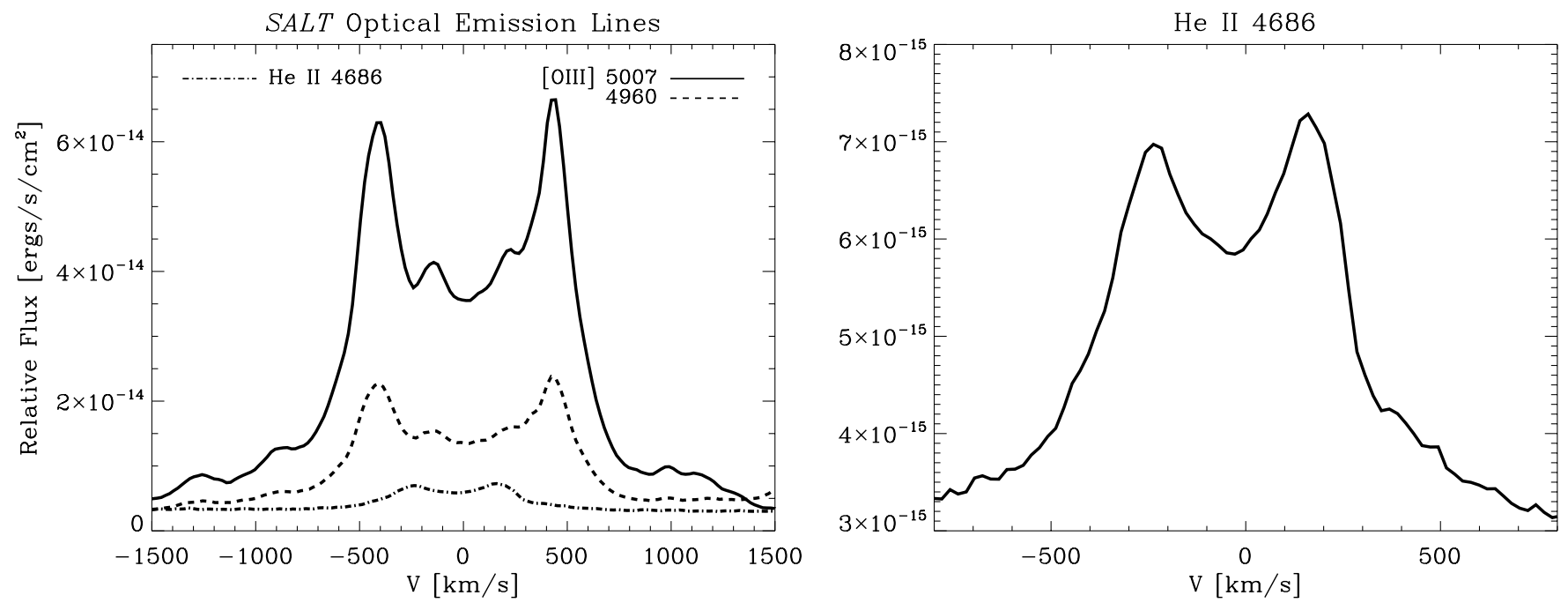

Figure 9. Left: SALT velocity spectrum of [O III] $\lambda \lambda 5007$ and 4960 and He II $\lambda 4686$ from the high-resolution spectrum of 2012 December 6 . Right: expanded velocity region around $\mathrm{He}$ II $\lambda 4686$ highlighting the difference in velocity structure of $\mathrm{He}$ II to [O III].

evidence of a late ejection of material around 100 days after the outburst, which may explain the different geometry of the He II lines or even the inner component of the [O III] lines.

In any case, the ejecta were not expanding with spherical symmetry even many months after the X-ray grating observations. Assuming that they were partially optically thick to soft $\mathrm{X}$-rays while occupying only a portion of the line of sight to the WD seems a reasonable conclusion.

\section{DISCUSSION}

Below we discuss five intriguing results of our analysis.

1. X-ray emission lines originate in dense clumps $\left(n_{e} \sim\right.$ $10^{10} \mathrm{~cm}^{-3}$ ) of cooling ejected plasma in CIE. The "soft" $\mathrm{X}$-ray emission line spectrum of T Pyx bears similarities with CNe V1494 Aql (Rohrbach et al. 2009) and V382 Vel (Ness et al. 2005), which showed strong emission lines over weak continuum at nearly the same time after the initial outburst as our T Pyx observations (268 days, V382 Vel; 304 days, V1494 Aql). Both were modeled as a plasma in $\mathrm{CIE}$ with enhanced $\mathrm{N}$ abundances and had an absence of $\mathrm{Fe}$ line emission. While these three novae all share unevolved companions, their outbursts were quite different outside of the X-ray regime. V382 Vel and V1494 Aql are very fast novae with decay times (time to decline 3 mag from peak) of $t_{3}=10$ days and $t_{3}=16$ days (Kiss \& Thomson 2000), respectively. T Pyx, for reference, has a decay time of $t_{3}=62$ days (Schaefer 2010).

We would like to also suggest a possible interpretation, that the spectra of these three novae above may be due to internal shocks from the interaction of different velocity shells within the ejecta (see Mukai \& Ishida 2001).

2. Many of the X-ray emission lines show blue shifted centroids that are largest at higher ionization potentials. Comparing the X-ray lines to the SALT nebular [O III] emission, which also trace ejected material from 602 days after outburst, it is clear that both an approaching and receding outflow exists that is centered near zero velocity (see Figure 9). At the time of our X-ray observations we note that the FWHM of the emission lines is much larger than their velocity offsets, which we interpret as a partial absorption in the receding flow of the hardest X-ray lines observed.
3. The continuum flux in the Chandra (day 210) X-ray spectra was best fit with a WD atmospheric model at a temperature of $\sim 420,000 \mathrm{~K}$. Assuming that this was the temperature at the peak of the SSS phase, theoretical models of nova outbursts for this atmospheric temperature predict a bolometric luminosity at, or above, $3 \times 10^{37} \mathrm{erg} \mathrm{s}^{-1}$ (Starrfield et al. 2012). This is of course a lower limit given that the fit temperature is likely less than at the outburst peak and indeed the nova may have turned off by the time of our observation. From our fit we derive $L_{\mathrm{Bol}}$ to be a factor of 10 less than predicted. Furthermore, even at the peak of X-ray emission (a $\sim 6$ times higher count rate; see Figure 1), the luminosity for the WD is still less than predicted.

The low luminosity observed, along with evidence of primarily observing the face of the ejected material, may point to an obscuration of the WD flux. A column density of $N_{\mathrm{H}} \sim 4 \times 10^{21} \mathrm{~cm}^{-2}$, or $\sim 3$ times our best-fit value, would be required to match the expected flux at this temperature. This column corresponds to an ejected mass of a few times $10^{-6} M_{\odot}$ assuming a filling factor of $3 \%$ and an outflow velocity of $4000 \mathrm{~km} \mathrm{~s}^{-1}$ (Shore et al. 2011). This seems a reasonable value but of course requires ejected mass to be along the line of sight.

It is also very puzzling that in the 25 days between X-ray grating observations, we observe no significant drop in the WD temperature but a factor of $>2$ decrease in continuum flux (see Figure 4). All acceptable model fits show that $N(\mathrm{H})$ does not seem to have increased drastically, which is what we expect at this stage. The rapid overall decrease in count rate (see Figure 1; Swift) is typical of the cooling curve, after nuclear burning has ceased, yet the WD does not appear to have cooled.

We see two possibilities to interpret this phenomenon: First, that the atmospheric model we have used (Rauch et al. 2010; hydrostatic atmosphere at a constant radius) may not be adequate to describe the X-ray spectra at this stage of the outburst. It is possible that the WD radius at the SSS peak was bloated due to a wind (see van Rossum 2012), so that the initial decay in the X-ray light curve is due to the wind ceasing and the WD radius finally shrinking to typical WD dimensions. In other words, if Rauch's models are correct, we expect a cooling at constant radius, whereas 
if van Rossum's “wind models" should be adopted, we expect first that the radius shrinks followed by a decrease in temperature. However, since van Rossum's models, so far, are only available with solar abundances (not typical of realistic novae) at this stage, we do not repeat the fit to the Chandra spectrum with these models.

Alternatively, Nelson et al. (2012) suggest a new episode of large, asymmetric mass ejection around day 100. Such material could decrease the flux while the WD temperature remained constant. We consider this possibility less likely at this late post-outburst stage, but it cannot be ruled out to explain what we observe in Figure 4 and the results of the atmospheric fit.

4. If the peak temperature did not vary significantly in the initial decline from the SSS phase, the short SSS turnoff time is unusual for an effective temperature $T_{\text {eff }}$ of the order of 400,000 K (Starrfield et al. 2012; see examples of other novae in Orio 2012, and references therein). This may indicate that more unburned H-rich envelope is left over here than in other novae. If a large unburned envelope is present, this nova does not need to accrete a large quantity of material to trigger a new outburst. It may quickly reach the pressure for TNR within a few years, a cycle that will continue, as long as the TNR events are all somewhat ineffective in ejecting the accreted envelope. Such a temporary "stunted" cycle of RN eruptions every $\simeq 20$ years may last until the WD manages to eject most of the unprocessed accreted material.

Schaefer et al. (2010) has suggested that an outburst prior to 1890 may have been the first after many centuries without eruption that, unlike the more recent events, was similar to a $\mathrm{CN}$ outburst. At that point $\mathrm{T}$ Pyx would initiate a phase of unsustainable irradiation induced mass transfer that, decaying over time, causes the present RN status to be transient in nature heading back toward an extended dormant state. If mass transfer was driven by an irradiated secondary, a high column density of the order of $10^{22} \mathrm{~cm}^{-2}$ would have been necessary in the month before the 2011 outburst to shield the hot source. The low pre-outburst softX-ray flux, the short-lived 2011 SSS phase, and the low temperature observed in T Pyx indicate that there is not a prolonged irradiation induced mass transfer after each RN outburst.

We would like to suggest a modification of the Schaefer et al. (2010) scenario, namely, that the frequency of the outbursts and the short, underluminous SSS duration indicate a nova that does not effectively expel its envelope in a single outburst. If T Pyx is an intermediate polar (IP), magnetic fields may be the cause of ineffective outbursts. Some analytical calculations were done by Livio et al. (1988), who, in the case of the stronger field of polars, find interference with convection, causing weaker outbursts than in a case with weak magnetic fields. On the other hand, a magnetic field may accelerate envelope expulsion once the TNR flash has occurred as in the magnetic rotator case analyzed by Orio et al. (1992), and thus ending a weak outburst quickly. For the time being this scenario is highly speculative, but we suggest that some unusual physical parameters are causing a long series of recurrent eruptions that do not eject the whole accreted envelope.

5. The orbital variability of the soft X-ray flux is not easy to interpret, but it is not unprecedented in $\mathrm{CNe}$. It was observed in HV Cet (Beardmore et al. 2012) and especially in V5116 Sgr (Sala et al. 2008), which for months showed a periodic decaying flare that Orio (2012) suggest may be due to resumed magnetic accretion that prevents the WD atmosphere from being thermally homogeneous. T Pyx may be a similar system in which renewed accretion streams to the polar caps prevent thermal homogeneity of the atmosphere. This conclusion is supported by the enhanced continuum flux of the elevated phase.

If T Pyx has a magnetic WD, the concurrent ephemeris with the optical light curve could be interpreted as an IP. In this case, the measured period would not be orbital in nature but instead the WD rotation period. This would require the WD spin to be $1.8 \mathrm{hr}$, making T Pyx the slowest rotating IP known (beyond EX Hya at $\sim 1.2 \mathrm{hr}$; King \& Wynn 1999). For a "disk-like" truncated accretion disk the ratio of spin to orbital period would have to be less than a tenth, but there are possibilities of "stream-like" and "ringlike" accretion structures on the equatorial plane, where the true orbital period would not have to be much longer, possibly only by a factor of $40 \%$ (Norton et al. 2008). The spectrum of the secondary should also otherwise be detected in quiescence, although in the case of CP Pup, Mason et al. (2013) hypothesize that an "accretion curtain" may mask the secondary's spectrum and go undetected. A relatively strong magnetic field of an IP may also explain why this nova is different from most others.

The above hypothesis is, however, at odds with the longterm increasing period observed by Patterson et al. (1998), which would require the WD to be spinning down if it were an IP (opposite the expectation of an accreting WD). An alternative scenario could involve obscuring material blown off the secondary during the outburst or modulation of an accretion disk structure coming into the line of sight. This would imply that the period we observe is indeed the orbital period. A partial obscuration scenario may, however, be at odds with the detection of the X-ray period in quiescence (Balman 2012) and would require the inclination to be larger than claimed in the current literature. Both explanations remain highly speculative but seem reasonable interpretations of the data.

\section{CONCLUSIONS}

In this paper, we have analyzed spectral and temporal components of X-ray grating and optical spectra during the decline of the 2011 outburst of RN T Pyx. Below we summarize the main findings of this work.

1. The X-ray grating spectra of T Pyx during the decline of the super-soft phase show a significant contribution from emission lines to the X-ray flux. These lines appear to be emitted from high-density clumps $\left(n_{e} \sim 10^{10} \mathrm{~cm}^{-3}\right)$ of ejected material in CIE. We interpret the blueshifted centroids of the lines as evidence of primarily seeing the face of the ejected material.

2. The X-ray continuum flux is modulated with commonly accepted orbital period.

3. We have not measured the peak $T_{\text {eff }}$ of the WD, and the nova may have in fact turned off before our observations. However, the fact that the temperature did not significantly vary between the Chandra and the XMM-Newton observations, while the overall continuum flux decreased, suggests that the initial decay occurred at an almost constant temperature, either because mass loss from a wind mechanism 
did not completely cease until weeks after the peak X-ray SSS luminosity, causing the initial decline to occur with a shrinking radius at near constant temperature, or because a new ejection of mass increased the amount of absorbing material along the line of sight (this seems less likely given the late post-outburst stage).

4. The luminosity we derive is more than an order of magnitude lower than expected and, even at the peak of the SSS flux, is at minimum a factor of $\sim 3$ too small. The low luminosity along with mainly blueshifted X-ray signatures from the ejecta suggest that the WD is partially obscured by the ejecta.

5. SALT spectra display nearly symmetric bipolar ejecta almost 2 yr after the outburst revealed by double-peaked emission lines of the [O III] doublet. He II $\lambda 4686$ and $\mathrm{H} \beta$ are also present, but with different velocity structures originating in either a reforming accretion disk or a separate small episode of mass ejection.

The authors would like to thank Richard Townsend for his aid in periodogram error analysis and Jay Gallagher for many useful discussions. We would also like to acknowledge the use of public data from the Swift data archive, and we acknowledge, with thanks, the variable star observations from the AAVSO International Database contributed by observers worldwide and used in this research. The Swift project at the University of Leicester is supported by the UK Space Agency. This work has been supported by a Chandra grant awarded by NASA through the Smithsonian Center for Astrophysics.

\section{REFERENCES}

Balman, S. 2012, arXiv:1208.6120

Beardmore, A. P., Osborne, J. P., Page, K. L., et al. 2012, A\&A, 545, A116 Blumenthal, G. R., Drake, G. W. F., \& Tucker, W. H. 1972, ApJ, 172, 205

Brinkman, A. C., Gunsing, C. J., Kaastra, J. S., et al. 1997, Proc. SPIE, 3113, 181

Buckley, D. A. H., Barnes, S. I., Burgh, E. B., et al. 2008, Proc. SPIE, 7014, 701407

Buckley, D. A. H., Swart, G. P., \& Meiring, J. G. 2006, Proc. SPIE, 6267, 62690A

Burgh, E. B., Nordsieck, K. H., Kobulnicky, H. A., et al. 2003, Proc. SPIE, 4841,1463

Cash, W. 1979, ApJ, 228, 939

Chesneau, O., Meilland, A., Banerjee, D. P. K., et al. 2011, A\&A, 534, L11

Churazov, E., Gilfanov, M., Forman, W., \& Jones, C. 1996, ApJ, 471, 673

Crawford, S. M., Still, M., Schellart, P., et al. 2010, Proc. SPIE, 7737, 773725

Downes, R. A., Duerbeck, H. W., \& Delahodde, C. E. 2001, JAD, 7, 6

Gehrels, N., Chincarini, G., Giommi, P., et al. 2004, ApJ, 611, 1005
Iben, I. J., \& Fujimoto, M. Y. 2008, in Classical Novae, ed. M. Bode \& A. Evans (2nd ed., Cambridge Astrophysics Series 43; Cambridge: Cambridge Univ. Press), 34

Kato, M., \& Hachisu, I. 1994, ApJ, 437, 802

King, A. R., \& Wynn, G. A. 1999, MNRAS, 310, 203

Kiss, L. L., \& Thomson, J. R. 2000, A\&A, 355, L9

Kobulnicky, H. A., Nordsieck, K. H., Burgh, E. B., et al. 2003, Proc. SPIE, 4841, 1634

Kraft, R. P., Burrows, D. N., \& Nousek, J. A. 1991, ApJ, 374, 344

Kuulkers, E., Page, K. L., Ness, J.-U., et al. 2011, ATel, 3285, 1

Livio, M., Shankar, A., \& Truran, J. W. 1988, ApJ, 330, 264

Mason, E., Bianchini, A., Orio, M., et al. 2013, MNRAS, 436, 212

Mukai, K., \& Ishida, M. 2001, ApJ, 551, 1024

Neff, J. S., Smith, V. V., \& Ketelsen, D. A. 1978, ApJS, 38, 89

Nelson, T., Chomiuk, L., Roy, N., et al. 2012, arXiv:1211.3112

Ness, J.-U., Schaefer, B. E., Dobrotka, A., et al. 2012, ApJ, 745, 43

Ness, J.-U., Starrfield, S., Jordan, C., Krautter, J., \& Schmitt, J. H. M. M. 2005, MNRAS, 364, 1015

Norton, A. J., Butters, O. W., Parker, T. L., \& Wynn, G. A. 2008, ApJ, 672, 524

Orio, M. 2012, BASI, 40, 333

Orio, M., Behar, E., Gallagher, J., et al. 2013, MNRAS, 429, 1342

Orio, M., Trussoni, E., \& Oegelman, H. 1992, A\&A, 257, 548

Osborne, J. P., Beardmore, A. P., Page, K. L., et al. 2011, ATel, 3549, 1

Patterson, J., Kemp, J., Shambrook, A., et al. 1998, PASP, 110, 380

Patterson, J., Oksanen, A., Monard, B., et al. 2013, arXiv:1303.0736

Porquet, D., \& Dubau, J. 2000, A\&AS, 143, 495

Porquet, D., Dubau, J., \& Grosso, N. 2010, SSRv, 157, 103

Porquet, D., Mewe, R., Dubau, J., Raassen, A. J. J., \& Kaastra, J. S. 2001, A\&A, 376, 1113

Rauch, T., Orio, M., Gonzales-Riestra, R., et al. 2010, ApJ, 717, 363

Ringwald, F. A., Naylor, T., \& Mukai, K. 1996, MNRAS, 281, 192

Robinson, E. L. 1976, ARA\&A, 14, 119

Rohrbach, J. G., Ness, J.-U., \& Starrfield, S. 2009, AJ, 137, 4627

Sala, G., Hernanz, M., Ferri, C., \& Greiner, J. 2008, ApJL, 675, L93

Scargle, J. D. 1982, ApJ, 263, 835

Schaefer, B. E. 2010, ApJS, 187, 275

Schaefer, B. E., Pagnotta, A., \& Shara, M. M. 2010, ApJ, 708, 381

Selvelli, P., Cassatella, A., Gilmozzi, R., \& González-Riestra, R. 2008, A\&A, 492, 787

Shore, S. N., Augusteijn, T., Ederoclite, A., \& Uthas, H. 2011, A\&A, 533, L8

Shore, S. N., Schwarz, G. J., De Gennaro Aquino, I., et al. 2013, A\&A, 549, A 140

Smith, R. K., Brickhouse, N. S., Liedahl, D. A., \& Raymond, J. C. 2001, ApJL, 556, L91

Snijders, M. A. J. 1990, in IAU Colloq. 122, Physics of Classical Novae, ed. A. Cassatella \& R. Viotti (Berlin: Springer), 188

Sokoloski, J. L., Crotts, A. P. S., Lawrence, S., \& Uthas, H. 2013, ApJL, 770, L33

Starrfield, S., Timmes, F. X., Iliadis, C., et al. 2012, BaltA, 21, 76

Strope, R. J., Schaefer, B. E., \& Henden, A. A. 2010, AJ, 140, 34

Szczerba, R. 1987, A\&A, 181, 365

Uthas, H., Knigge, C., \& Steeghs, D. 2010, MNRAS, 409, 237

van Rossum, D. R. 2012, ApJ, 756, 43

Waagan, E., Linnolt, M., \& Pearce, A. 2011, IAUC, 9205, 1

Webbink, R. F., Livio, M., Truran, J. W., \& Orio, M. 1987, Ap\&SS, 131, 493

Yaron, O., Prialnik, D., Shara, M. M., \& Kovetz, A. 2005, ApJ, 623, 398 